\title{
问

2-26-2015

\section{Taming the Unknown: A History of Algebra from Antiquity to the Early Twentieth Century}

Calvin Jongsma

Dordt College, calvin.jongsma@dordt.edu

Follow this and additional works at: https://digitalcollections.dordt.edu/faculty_work

Part of the Mathematics Commons

\section{Recommended Citation}

Jongsma, C. (2015). Taming the Unknown: A History of Algebra from Antiquity to the Early Twentieth Century. MAA Reviews Online Retrieved from https://digitalcollections.dordt.edu/faculty_work/217

This Book Review is brought to you for free and open access by Dordt Digital Collections. It has been accepted for inclusion in Faculty Work Comprehensive List by an authorized administrator of Dordt Digital Collections. For more information, please contact ingrid.mulder@dordt.edu. 


\title{
Taming the Unknown: A History of Algebra from Antiquity to the Early Twentieth Century
}

\author{
Abstract \\ Reviewed Title: Katz, Victor J. and Karen Hunger Parshall. Taming the Unknown: A History of Algebra from \\ Antiquity to the Early Twentieth Century. Princeton, NJ: Princeton University Press, 2014. 485 pages. ISBN \\ 9780691149059 \\ Keywords \\ book review, Victor J. Katz, Karen Hunger Parshall, Taming the Unknown, algebra \\ Disciplines \\ Mathematics \\ Comments \\ - http://www.maa.org/publications/maa-reviews/taming-the-unknown-a-history-of-algebra- \\ from-antiquity-to-the-early-twentieth-century \\ - Copyright (c) Mathematical Association of America, 2015.
}




\section{Taming the Unknown: A History of Algebra from Antiquity to the Early Twentieth Century}

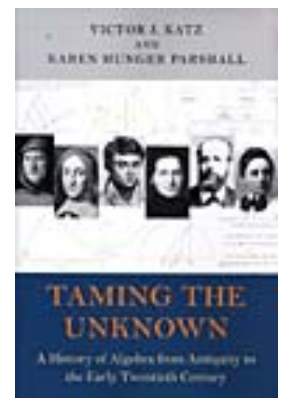

MAA REVIEW
Victor J. Katz and Karen Hunger Parshall

Publisher: Princeton University Press

Publication Date: 2014

Number of Pages: 485

Format: Hardcover

Price: $\quad \$ 49.50$

ISBN: $\quad 9780691149059$

Category: Monograph

\section{[Reviewed by Calvin Jongsma, on 02/26/2015]}

The prominent (and recently deceased) historian of mathematics Ivor Grattan-Guinness observed with regret back in the 1990s that "the history of mathematics is a classic example of a ghetto subject: too mathematical for historians and too historical for mathematicians." To which he added that it was "both too historical and too mathematical for modern professionalized philosophers" and that "specialists in mathematical education are little more inclined to take history seriously than are professional mathematicians." One might wonder where that leaves the audience for Taming the Unknown: A History of Algebra from Antiquity to the Early Twentieth Century.

There are signs, however, that indifference to the history of mathematics among North American mathematicians and mathematics educators has softened somewhat in the intervening decades: in addition to professional activities and journals devoted to history of mathematics, there is the thriving special interest group HOMSIGMAA, founded in 2001; history of mathematics sessions and short courses regularly offered at the Joint Meetings, MathFest, and other mathematical conferences; MAA's online journal Convergence, devoted since 2004 to providing teachers with educational resources in the history of mathematics; and books and articles discussing various aspects of the history of mathematics for mathematics educators. History of mathematics may still not be required everywhere for mathematics majors (it is at my institution), but there does seem to be increased interest from a number of mathematics educators who want to incorporate some degree of historical information into the courses they teach.

Instructors are often curious about when a certain result or technique was first discovered or about what major changes took place in the field that gave rise to what we have today. This latter concern is especially keen with regard to algebra, because while this field includes both classical algebra (studied in middle and high schools) and abstract algebra (studied in colleges/universities and graduate schools), these two areas seem to have little in common. Are they really connected by something more than a 
name? Katz and Parshall, following Grattan-Guinness, label such backward-looking interest as a concern with mathematical heritage. And while this is not the focus of history, which traces formative developments within particular socio-cultural contexts forward from the problems, methods, and outlooks inherited from the past, a knowledge of history can certainly contribute to a deepened understanding of a field as it is presently configured. With respect to teaching and learning mathematics, it can provide motivation and enrichment, as well as insight into both content and pedagogy.

Katz and Parshall have actively pursued and promoted the history (and heritage) of mathematics for decades, professionally as historians and also as mathematical educators. This book is their latest offering. As they note in their Prelude (Chapter 1), over the last few decades there have been a number of good articles and monographs on particular time periods, cultures, and aspects of the history of algebra, and there has even been some attempt at popularizing this material, but we still have no work that comprehensively synthesizes and organizes up-to-date research on the topic to make it accessible to mathematicians and mathematics educators. Until now.

Any history of algebra needs to take a stand on what algebra comprises, either via an explicit definition or by what it tacitly decides lies within its purview. Taming the Unknown verges on giving its readers a definition of "algebra," but in the end shies away from being too prescriptive, for good reasons. Contemporary understandings, whether aligned with elementary algebra (solving equations for unknowns, using a computational notation) or modern abstract algebra (the abstract study of operational and relational structures, like groups and lattices), invariably miss aspects associated with the other one. And, in fact, both sorts fail to provide a description that would encompass a work such as al-Khwārizmī's ninth-century al-Jabr, from which the term 'algebra' derives, or even earlier work in algebraic problem solving done by Egyptian, Babylonian, Greek, Chinese, and Indian cultures.

Katz and Parshall do venture to give us a periodization of algebra, however. The standard older classification is due to Nesselman (1842), who categorized historical developments in algebra in terms of their symbolic form (rhetorical; syncopated; fully symbolic). Taming the Unknown recognizes this to be deficient: algebra involves much more than symbolic forms of representation, so why should that be our sole criterion for distinguishing periods? It proposes instead a four-stage periodization: 1) a concrete geometric stage; 2) a static, equation-solving, algorithmic stage; 3) a dynamic stage, typified by an operative symbolism, equations for curves, and applications to physics; and 4) a final abstract stage, in which a variety of different algebraic structures and number systems are studied in their own right.

This classification links to a much broader range of concerns than the older one, but it still needs some further refinement: their geometric stage was already highly algorithmic - quantitative solutions were arrived at by following step-by-step procedures, even if geometric figures were manipulated, and (as they acknowledge) not all ancient algebraic practices seem to have passed through such a stage; their algorithmic stage continued to be constrained to a large degree by geometric considerations, even as late as Cardano and Viète; and their dynamic stage lacks a certain coherence, as not everything there is tied to treating dynamic phenomena. Nevertheless, their classification recognizes some important changes in algebra as they arise historically and come to typify the field in its new forms, which may be better than trying to identify a timeless essence characterizing the entire field.

Regardless of the limitations of the classification scheme put forward, a more important consideration is how Taming the Unknown narrates significant historical developments in algebra. Here the book is on solid ground. While historians might desire some additional cultural and intellectual context, mathematicians and mathematics educators will find a wealth of technical material of interest to them on the development of algebra over all time periods and places.

The first half or so of Taming the Unknown focuses on various pre-modern algebraic practices and theories, those occurring in Egyptian, Mesopotamian, Greek, Chinese, Indian, Arabic, and Medieval and Renaissance European cultures. This covers the authors' first two stages in the history of algebra. Greek 
and Indian cultures contained a number of geometric results that some have controversially termed "geometrical algebra," since when its results are represented in modern notation they become algebraic identities. Old Babylonian algebra, while recorded on tablets that simply present algorithms for solving a variety of what we might call first- and second-degree problems, is a genuinely geometrically-based algebra, using rectangle cut-and-paste procedures to arrive at solutions. Ancient Egyptian algebra, though very early, lacks any obvious geometric connections; Katz and Parshall tie this instead to an interest in proportionality. Later Greek algebra (Katz and Parshall devote a full chapter to exploring the riches of Diophantus' Arithmetica with its indeterminate problem types and solutions) and Medieval Indian algebra also seem independent of any geometrical sub-basis, manipulating algebraic expressions in formulaic ways to solve equations. Ancient Chinese algebra likewise lacks a geometric basis; it contributes computational ways of solving systems of first-degree problems, both by using a double falseposition technique and by using matrix elimination methods. They solved higher-degree polynomial equations using numerical approximation techniques.

Medieval Islamic algebra, identified as initiating the algorithmic stage, is the most immediate source of later European algebra. Here algebra becomes a more systematic discipline. Al-Khwārizmī delineates solution algorithms for the six basic canonical forms of first- and second-degree equations; he also introduces calculations with (verbal) algebraic expressions for putting more complex equations into standard formats. Geometry functions for him similarly to how it did for Mesopotamian scribes: as a rationale for legitimizing his solution algorithms. Later writers who became familiar with the geometrical algebra they found in Euclid sought to make that into a more rigorous deductive basis for those algorithms. Conic sections come into play in the algebra of al-Khayyāmī, who around 1100 was able to solve all fourteen types of cubic equations geometrically using intersections of such figures.

A number of medieval reckon-masters, beginning with Fibonacci, included translations/paraphrases from Arabic texts in some of their commercial arithmetics, and some attempted to extend this to solve higher degree equations. Little genuine success was had, though, until the 16th century, when (as is wellknown) Renaissance mathematicians del Ferro, Tartaglia, Cardano, and Ferrari discovered how to solve cubic and quartic equations algebraically. During this time period, mathematicians also began to use a variety of abbreviations for the various quantities and operations appearing in their equations.

By the end of the 16th century with the work of Viète and especially during the first half of the 17th century in the work of Harriot, Fermat, and Descartes, mathematicians began to treat algebra more symbolically, eventually adopting a notation that readily lends itself to making algebraic computations. This signals the start of Katz and Parshall's third stage of algebra. Having letters stand both for knowns and unknowns finally allowed for the possibility of presenting solutions to equations as compact general formulas. This is also the time period when letters began to represent variables instead of unknowns to be solved for, so that connections could be made between equations and curves in the analytic geometries of Fermat and Descartes. And, as the 17th and 18th centuries progressed, mathematicians and natural scientists began to use mathematical formulas to represent a wide variety of quantitative dynamic relationships. Analysis was extended from its more ordinary algebraic beginnings into the realms we know as calculus and differential equations, where solutions to equations could be functions and curves as well as numbers.

An interest in finding elementary methods for solving higher degree equations continued during this time period. Progress was made toward the end of the 18th century in the work of Lagrange by using permutations of the roots of an equation. Ruffini and Abel took these ideas further in demonstrating that fifth degree equations were in general unsolvable using radicals. Gauss's investigation of the solutions of cyclotomic equations also revealed group theoretic connections. But the mathematician who applied the notion of a group of permutations (in conjunction with the emerging notion of a field) most pervasively and successfully to solving algebraic equations was the short-lived Galois. By the time his work on this became better known and appreciated (solidly by 1870), mathematicians had begun to recognize the 
importance of groups in algebra and elsewhere.

The transition to a more abstract structured approach to algebra (stage four) took place gradually during the nineteenth century and was fed by a number of streams. Besides being used in solving higher degree equations, algebraic structures such as groups, fields, rings, and ideals arose in connection with number theory (modular arithmetic, quadratic forms, work on Fermat's Last Theorem), geometry (Klein's Erlanger Programm, Lie's continuous transformation groups), linear algebra (determinants, linear transformations, matrices, vectors), an expanding number concept (Gaussian and cyclotomic and algebraic integers, complex numbers, quaternions), and more focused treatments of groups (permutation groups, abstract groups). In addition to all this, mid-century developments in Great Britain advocated a more abstract approach to algebra, which promoted and supported some of the other trends. The history of algebra during this time period is too rich a tapestry to summarize adequately here, but Katz and Parshall flesh out this story over several chapters with suitably detailed exposition of the mathematics and the historical connections.

The book ends with a brief chapter highlighting some developments that established the new axiomatic approach to algebra as a theory of abstract structures, work by Peano, Hilbert, Fraenkel, Steinitz, Emmy Noether, and others. The story told by Taming the Unknown concludes around 1930, when the new approach was institutionalized through the publication of the seminal two-volume textbook Moderne Algebra by van der Waerden. Here the program we now recognize as typifying modern abstract algebraic concerns - the study of a variety of algebraic structures with their substructures; homomorphisms between structures; construction of new structures from old ones using products, residue classes, and quotient constructions - was expounded in architectonic fashion. This work became the standard for all later abstract algebra texts, whatever their distinctive features.

To sum up, Taming the Unknown provides an excellent and wide-ranging historical resource for those of us who teach algebra courses on different educational levels, from middle school through university, or just for those who would like to know how the different incarnations of algebra are related. The authors include numerous quotes from the primary actors in the drama, but they also provide helpful commentary (with reformulation into contemporary notation, where they deem it appropriate) so one can follow the train of thought and connections being presented. As the book contains twenty-seven pages of bibliographic references, one can easily follow up further on any topic of interest. Readers may occasionally find an aspect of the work they disagree with or that they wish was treated differently (I would have liked to have seen Boole discussed for his more important work on the algebra of logic than for his contribution to invariant theory), but overall there is much here to appreciate and learn from. Katz and Parshall address a significant need and put teachers of mathematics in their debt. Whether or not one buys a personal copy of this book (it's relatively inexpensive compared to a mathematics text), it should certainly find a place in every academic library.

Calvin Jongsma received his $\mathrm{Ph}$. $\mathrm{D}$. in mathematics/history of mathematics from the University of Toronto. He is now a semi-retired Professor of Mathematics at Dordt College in Sioux Center, IA. His email address is Calvin.Jongsma@dordt.edu.

\footnotetext{
Tags: $\quad$ Algebra

Classical Algebra

History of Mathematics

Log in to post comments
} 
P: (800) 331-1622

F: (240) 396-5647

E: maaservice@maa.org

Copyright () 2015 